\title{
RETRACTED ARTICLE: Significance of Increased Serum Interleukin-18 Levels in Stroke Patients: A Meta-Analysis
}

\author{
Yong Hao • Qiang Hu • Xiao-Jie Zhang • Jian-Qi Xiao • \\ Ze-Zhi Li • Yang-Tai Guan
}

Received: 22 September 2014 / Accepted: 30 October 2014 / Published online: 17 August 2015

(C) Springer Science+Business Media New York 2014

The Publisher and Editor retract this article in accordance with the recommendations of the Committee on Publication Ethics (COPE). After a thorough investigation we have strong reason to believe that the peer review process was compromised.

The original article was published online on November 26, 2014.

Y. Hao $\cdot$ Z.-Z. Li $(\bowtie) \cdot$ Y.-T. Guan $(\bowtie)$

Department of Neurology, Shanghai Changhai Hospital, Secondary

Military Medical University, No. 168 Changhai Road,

Shanghai 200433, People's Republic of China

e-mail: lizezhi1981@aliyun.com

e-mail: yangtaiguan@hotmail.com

Q. Hu

Department of Psychology, Qiqihar Mental Health Center,

Qiqihar 161000, People's Republic of China

X.-J. Zhang

Department of Pathology, Qiqihar Medical University,

Qiqihar 161000, People's Republic of China

J.-Q. Xiao

Department of Neurosurgery, The First Hospital of Qiqihar City,

Qiqihar 161000, People's Republic of China 